\title{
Production sources and food web structure of a temperate tidal estuary: integration of dietary and stable isotope data
}

\author{
Kirk O. Winemiller ${ }^{1, *}$, Senol Akin ${ }^{1,2}$, Steven C. Zeug ${ }^{1}$ \\ ${ }^{1}$ Ecology and Evolutionary Biology, Department of Wildlife and Fisheries Sciences, Texas A\&M University, 2258 TAMU, \\ College Station, Texas 77843-2258, USA \\ ${ }^{2}$ Present address: Faculty of Agriculture, Department of Fisheries, Gaziosmanpasa University, 60240 Tokat, Turkey
}

\begin{abstract}
Food web structure and major sources of primary production consumed by metafauna of Mad Island Marsh, a coastal saltmarsh on the NW coast of the Gulf of Mexico, were compared using stable isotopes and dietary analysis. Carbon and nitrogen isotope data were entered into a mixing model containing 5 potential production sources. Results were inconclusive due to overlapping isotopic signatures of certain sources, but nonetheless indicated that most fishes and macroinvertebrates assimilated material derived mostly from variable mixtures of macrophytes and filamentous algae. Highest estimates of percentage of material assimilated directly or indirectly from $\mathrm{C}_{4}$ marsh grasses (ranging from 30 to $82 \%$ ) were for spot Leiostomus xanthurus and Gulf killifish Fundulus grandis. Isotopic analysis could not reveal the detailed structure of predator-prey interactions at the species level; greater detail of trophic pathways was revealed by the dietary analysis. Estimates of vertical web structure (species trophic levels) by the 2 methods were largely concordant. The exceptions were 2 zooplanktivorous and detritivorous fish species and grass shrimp Palaemonetes pugio that had higher trophic levels according to nitrogen isotope ratios. For these taxa, the isotopic method more accurately indexed the number of trophic transfers than the dietary method, which depends on accurate dietary estimation for all food chain components leading to a consumer, and which assumes equal assimilation efficiencies for items found in stomach contents. The isotopic method underestimated trophic levels of several invertebrates, possibly due to inaccurate estimation of mean $\delta^{15} \mathrm{~N}$ for production sources supporting these taxa and/or differential trophic fractionation. Together, stable isotope and dietary analyses provide a more accurate assessment of food web structure and dynamics of coastal marsh ecosystems than either method alone.
\end{abstract}

KEY WORDS: Carbon · Cordgrass · Detritivory · Estuary · Gulf of Mexico · Nitrogen · Predation · Trophic structure

Resale or republication not permitted without written consent of the publisher

\section{INTRODUCTION}

Food webs - networks of species and trophic pathways - provide a holistic yet detailed description of ecosystems and community interactions (Cohen et al. 1990, Winemiller \& Polis 1996). When species and pathways are quantified, food webs also provide a basis for modeling the dynamics of community elements (Baird \& Ulanowicz 1989, Christensen \& Pauly 1992, Yodzis 1998). Empirical estimation of species abundances and the strengths of species interactions is a major challenge for food web research (Berlow et al. 2004, Winemiller \& Layman 2005). Here we report empirical estimates of food web structure and production sources supporting consumer taxa of a tidal saltmarsh ecosystem along the NW Gulf of Mexico coast. We then compare characteristics of this food web with those from earlier studies on coastal marshes as well as other kinds of aquatic and marine ecosystems. 
Many studies have identified feeding patterns of estuarine consumer species (Diener et al. 1974, Sheridan 1978, Divita et al. 1983, Matlock \& Garcia 1983, Scharf $\&$ Schlicht 2000), but very few have examined more than a few species within a local community. A great deal of research has examined sources of primary production supporting estuarine consumers (Haines \& Montague 1979, Fry \& Sherr 1984, Currin et al. 1995, Kwak \& Zedler 1997, Bouillon et al. 2002, Sobczak et al. 2002, Connolly et al. 2005). Many earlier estuarine studies identified detritus as the principal production source for estuarine food webs, with the sources of detritus consumed by microbes and metazoans varying among locations and seasons (Odum et al. 1973, Peterson \& Howarth 1987, Coffin et al. 1989, Deegan \& Garritt 1997). Based on results from studies of other North American Atlantic and Gulf coast estuaries (e.g. Marinucci 1982, Peterson \& Howarth 1987, Sullivan \& Moncreiff 1990, Weinstein et al. 2000, Sobczak et al. 2002), we hypothesized that the dominant aquatic primary producers, saltmarsh grasses Spartina spp. and algae (phytoplankton and benthic microalgae), are the principal sources of carbon and energy for the Mad Island Marsh food web. Using volumetric stomach contents analysis, Akin \& Winemiller (2006) examined seasonal variation in food web structure of Mad Island Marsh, a small tidal estuary on the SW Gulf of Mexico coast. Seasonal variation in web properties was low, with detritus being a dominant food category in diets of macrofauna, a finding consistent with earlier studies of Gulf estuarine communities (e.g. Darnell 1961). Low variation in web structure occurred despite seasonal changes in the fish species assemblage and size structure of several resident populations.

Quantitative models of estuarine food webs have relied on compilations of literature information from diverse taxa and regions, often involving coarse extrapolations (Baird \& Ulanowicz 1989, ManickchandHeileman et al. 1998). Herein, we investigated species trophic relationships at Mad Island Marsh using 2 methods: dietary and stable isotope analysis. Diets were estimated as the proportional volumetric consumption of food items recovered from stomachs of fishes and decapod crustaceans. Stable isotope methods exploit patterns of fractionation for ratios of heavy and light isotopes of carbon and nitrogen in organic material pools. We compared estimates of potential primary production sources supporting macrofauna and vertical trophic structure based on analysis of dietary versus isotopic data. Based on findings from similar research on a stream community (Mantel et al. 2004) and several estuarine species (e.g. Creach et al. 1997), we anticipated that results from isotope methods and dietary analysis would complement each other to yield a more robust model of food web structure and dynamics.

\section{MATERIALS AND METHODS}

Study area. Mad Island Marsh (MIM) is located on the fringe of Matagorda Bay, Texas on the NW coast of the Gulf of Mexico (a more detailed description of environmental conditions, temporal/spatial variation, and a map appear in Akin et al. 2003). Aquatic habitats in MIM span physiochemical gradients from freshwater, in Mad Island Slough and diked wetlands, to an oligo-mesohaline lake and surrounding saltmarsh, to a polyhaline tidal bayou. Our study was restricted to the brackish lake, fringing saltmarsh, and the tidal bayou that connects the lake to the bay (6 survey sites arranged along a $3 \mathrm{~km}$ longitudinal depth/salinity gradient, see map in Akin et al. 2003). Salinity ranged from 3.5 to 29 psu (most values 5 to 20 psu), with highest values recorded during late summer in the tidal bayou, and lowest values during winter in the uppermost site of the lake. The area (2307 ha) is managed by the Nature Conservancy of Texas, which has a restoration program that conducts controlled burns and augments freshwater inflow to restore and enhance marsh habitat. Dominant vegetation of the marsh includes cordgrasses Spartina alterniflora, S. patens, S. spartinae and rush sedges Juncus spp. Fringing uplands are dominated by seacoast bluestem Schizachyrium scoparium, Gulf dune paspalum Paspalum monostachyum, glasswort Salicornia bigelovii, saltwort Batis maritima, sea oxeye daisy Borrichia frutescens, marsh elder Iva frutescens, and exotic Bermuda grass Cynodon dactylon. Submerged aquatic vegetation, such as widgeon grass Ruppia maritima and musk grass Chara sp., is abundant during warm months, especially within the upper portion of the estuary. The macroalga Ulva sp. occurs sporadically in patches with low biomass. Aquatic habitats of the study reach are shallow ( 0.10 to $0.75 \mathrm{~m})$, with substrata consisting of a mudsand mixture, covered by an approximately $5 \mathrm{~cm}$ layer of decomposing vegetation. Small patches of oyster reef and crushed oyster were present in the 3 sites closest to the bay. Diurnal tidal flux at MIM typically is low $(0.10$ to $0.25 \mathrm{~m}$, with $0.5 \mathrm{~m}$ infrequently), and largest variation in water depth is associated with local precipitation (Gelwick et al. 2001).

Sample collection. Water depth, temperature, dissolved oxygen, salinity, and the percentage of water surface area covered by mats of submerged plants (Chara sp. and Ruppia maritima) were estimated at each survey site every 2 mo from March 1998 through August 1999 to represent environmental variation at the time of biological sampling. These environmental results are presented elsewhere (Akin et al. 2003). A metal drop-sampler was used to quantify the density of benthic invertebrates and macrophytes enclosed within an area of $0.09 \mathrm{~m}^{2}$. The sampler had no bottom and 
was $1.0 \mathrm{~m}$ high. The sampler was operated by hand and dropped on an undisturbed area. Using a finemesh dipnet, macrophytes and sediment within the core were collected, the latter to a depth of $10 \mathrm{~cm}$. Samples were then placed into a 501 plastic bag and preserved in $10 \%$ formalin with rose-bengal solution that stains chitin to aid invertebrate sorting. In the laboratory, fine sediments were rinsed through a $0.25 \mathrm{~mm}$ mesh sieve, and preserved invertebrates were then sorted, identified, and counted.

Except for gillnet samples, specimens were collected between 09:00 and 16:00 h. For zooplankton samples, 51 of water were obtained from the water column using a plastic bottle that was then placed on ice and transported to the laboratory for analysis. Experimental gillnets $(38 \times 2 \mathrm{~m}$ with 5 panels of $25,38,50,64$, and $76 \mathrm{~mm}$ mesh) and a bag seine $(6 \times 1.2 \mathrm{~m}, 4.5 \mathrm{~mm}$ mesh, and bag $1.2 \times 1.2 \mathrm{~m}$ ) were used to sample fishes, crabs, and shrimp. For a given site survey, seining continued until no additional species were encountered in 3 consecutive hauls. To calculate catch per unit effort (CPUE as individuals or biomass $\mathrm{m}^{-1}$ seined) for species, the number of hauls and the distance of each haul were recorded. Monofilament experimental gillnets were deployed at all sites within $1 \mathrm{~h}$ of each other, with nets deployed from ca. 08:30 to 21:30 h. Duration of each gillnet set was recorded, and catch data were standardized as number of individuals captured $\mathrm{h}^{-1}$. Captured fishes and invertebrates were anesthetized in MS-222 and then fixed in 10\% formalin in the field. In the laboratory, preserved specimens were identified to species, measured (standard length, SL, to nearest $0.1 \mathrm{~mm}$ ), and weighed (to nearest $0.1 \mathrm{~g}$ ).

For stable isotope analysis, samples of sediment, vegetative detritus, phytomicrobenthos with sediment organic matter (SOM), phytoplankton and other suspended particulate matter (SPM), filamentous algae, macroalgae (Chara sp., Ulva sp.), and vascular plants were collected from Sites 1 to 3 during August 1999. Any macrophyte tissues with epiphytic algae were scrubbed by hand and rinsed with distilled water. Samples were also collected during February, but these samples yielded no data due to problems during mass spectrometry. Sediment samples consisting of clay and associated organic material and microorganisms were obtained with a corer to a depth of $10 \mathrm{~cm}$, placed in bags, and frozen. Vegetative detritus was collected from the top of the undisturbed lake bottom with a hand net, rinsed, placed in bags, and frozen. Samples of the top 1 to $2 \mathrm{~mm}$ layer of fine organic sediment, microalgae, and microorganisms (referred to here as phytomicrobenthos/SOM) were obtained with a small spatula, placed in plastic bags, and frozen. Samples from mats of filamentous algae growing on firm substrates (oyster shells, Spartina sp. stems) were obtained with a small spatula, rinsed, placed in plastic bags, and frozen. Samples from mats of macroalgae were collected by hand, rinsed with estuary water to remove associated organisms, placed in plastic bags, and frozen. Leaves of dominant terrestrial and aquatic vascular plants were removed, placed in plastic bags, and frozen.

Water samples were collected in 21 plastic bottles that were maintained on ice for transport to the laboratory, where they were filtered through a $100 \mu \mathrm{m}$ seive to obtain samples of zooplankton. Three representative subsamples taken from the zooplankton sample were examined under a light microscope to reveal the presence of phytoplankton. When zooplankton samples were confirmed to be free of phytoplankton, distilled water was added for easy filtering of the contents through ashed $\left(450^{\circ} \mathrm{C} \times 4 \mathrm{~h}\right) 47 \mu \mathrm{m}$ glass filters $(\mathrm{GF} / \mathrm{F})$. Each filter with zooplankton was dried at $60^{\circ} \mathrm{C}$ for $2 \mathrm{~d}$ in an oven. Zooplankton-free water samples were then stirred, and subsamples were examined for the presence of organisms or materials other than phytoplankton. Distilled water was then added to samples verified to be zooplankton-free. Each of these samples was then passed through a $27 \mu \mathrm{m} \mathrm{GF/F} \mathrm{filter} \mathrm{to} \mathrm{obtain} \mathrm{a}$ sample of particulate organic matter assumed to be dominated by phytoplankton (referred to hereafter as 'phytoplankton/SPM').

Tissue samples for isotope analysis of macroinvertebrates and fishes were collected from Sites 1 to 3 during August 1999. A few samples were collected at other times - February (all Gulf menhaden Brevoortia patronus, 1 pinfish Lagodon rhomboides, 3 silver perch Bairdiella chrysoura, and 1 Gulf killifish Fundulus grandis), April (2 black drum Pogonias cromis and 2 southern flounder Paralichthys lethostigma), and June (10 striped mullet Mugil curema, 3 ladyfish Elops saurus, 1 red drum Sciaenops ocellatus). Muscle tissue of oysters and mussels (adductor), crabs (chela), and shrimp (tail) was excised, rinsed in distilled water, and frozen in plastic bags. Because grass shrimp Palaemonetes pugio were small, each isotopic sample combined tail muscle tissue from 10 individuals. Muscle/ skin tissue samples (approximately $5 \mathrm{~g}$ ) were removed from the dorsum of large fish specimens $(>3 \mathrm{~cm} \mathrm{SL}$ ) for processing, whereas the entire specimen (minus the gut) was collected and processed for small size classes $(<3 \mathrm{~cm})$. For the smallest fishes (e.g. juvenile bay anchovy Anchoa mitchilli and Gulf menhaden, SL $<1.5 \mathrm{~cm}$ ), 10 individuals were pooled as a single sample. Samples for isotopic analysis were placed in plastic bags and then frozen.

Stomach contents analysis. Stomach contents analysis was performed to quantify consumer diets according to methods described in Winemiller (1990). When sufficient numbers of specimens were available in a 
given survey sample, at least 30 specimens of each fish species and 3 to 5 specimens of each macrocrustacean species (shrimp and crabs) were dissected for analysis. When a sample contained $>30$ specimens, individuals were selected for dissection so that size classes were represented in proportions approximating those in the field sample. Because piscivores frequently had empty stomachs, all available specimens of piscivores were examined for stomach contents. Ultimately, species sample sizes for stomach contents analysis ranged from 1 (5 rare species) to 910 (bay anchovy) (mean = $115, \mathrm{SD}=191$ ). All food items were removed from the anterior half of the gut and examined under a dissecting microscope, or compound microscope, depending on prey size.

Prey items were recorded based on 130 categories with variable levels of taxonomic aggregation, ranging from species to orders and functional groups, such as detritus and filamenous green algae. Small invertebrates, such as microcrustacea and insects were classified to taxonomic order. Fishes and macrocrustaceans, such as grass shrimp, brown shrimp Farfantepenaeus aztecus, white shrimp Litopenaeus setiferus, blue crab Callinectes sapidus and mud crabs Neopenope sayi and Eurypanopeus debressus, were classified to species level. For larger prey items ( $>0.1 \mathrm{ml})$, the entire sample was blotted dry with a paper towel then measured in a graduated cylinder by water displacement. For volumes less than $0.1 \mathrm{ml}$, volume was visually estimated by placing the item on a glass slide and comparing it with a known volume of water dripped from a pipette.

Detritivores and algivores were handled differently from species that consume individual food particles. The entire gut of each detritivore was removed and a small sample of its contents from the foregut was placed on a glass slide for examination under a light microscope. The volume of the entire gut and its contents then was measured by water displacement. The relative proportion of each identifiable item on the slide was estimated visually, and then multiplied by the volume of the gut plus contents, as determined by water displacement.

Sample processing for isotopic analysis. Fish and macroinvertebrate tissue samples were thawed, soaked and rinsed in distilled water, then dried in an oven at $60^{\circ} \mathrm{C}$ for $48 \mathrm{~h}$. Dried samples were ground to a fine powder with a pestle and mortar then stored in clean glass vials. Macrophyte, macroalgae, and filamentous algae samples were rinsed with distilled water, dried at $60^{\circ} \mathrm{C}$ for $48 \mathrm{~h}$, and ground to a fine powder. Phytoplankton/SPM and phytomicrobenthos/ SOM samples were filtered through pre-combusted $\left(450^{\circ} \mathrm{C}\right.$ for $\left.24 \mathrm{~h}\right) \mathrm{GF} / \mathrm{C}$ filters. Filters with organic material were then dried at $60^{\circ} \mathrm{C}$ for $48 \mathrm{~h}$. Zooplankton samples were filtered through GF/C filters. Each filter was then placed under a compound microscope, and any obvious animals (mostly copepods) were gathered from the filter using fine forceps. These zooplankton were placed in distilled water and then filtered through pre-combusted $\left(450^{\circ} \mathrm{C}\right.$ for $24 \mathrm{~h}$ ) GF/C filters and dried. Sediment core samples were soaked for $24 \mathrm{~h}$ in dilute $\mathrm{HCl}$ then soaked and rinsed with distilled water before drying.

Samples were analyzed for stable isotope ratios $\left({ }^{13} \mathrm{C} /{ }^{12} \mathrm{C}\right.$ and $\left.{ }^{15} \mathrm{~N} /{ }^{14} \mathrm{~N}\right)$ at the Analytical Chemistry Laboratory, Institute of Ecology, University of Georgia, Athens. Subsamples for each sample were weighed to $10^{-6} \mathrm{~g}$, pressed into Ultra-Pure tin capsules (Costech), then dry-combusted (micro Dumas technique) with a Carlo Erba CHN elemental analyzer. Purified gases $\left(\mathrm{CO}_{2}\right.$ and $\left.\mathrm{N}_{2}\right)$ were introduced into a Finnigan Delta $\mathrm{C}$ mass spectrometer, and the isotopic composition was quantified relative to a standard reference material. Standards were carbon in the PeeDee Belemnite and molecular nitrogen gas in the air. Results were reported as parts per mille (\%) differences from the corresponding standard:

$$
\delta X=\left[\left(\mathrm{R}_{\text {sample }} / \mathrm{R}_{\text {standard }}\right)-1\right] \times 10^{3}
$$

where $\mathrm{R}={ }^{15} \mathrm{~N} /{ }^{14} \mathrm{~N}$ or ${ }^{13} \mathrm{C} /{ }^{12} \mathrm{C}$.

Data analysis. Estimation of assimilated basal source material from isotopic data: In order to identify the potential range of basal source contributions for aquatic consumers, the Isosource routine described by Phillips \& Gregg (2003) was performed using a 2-isotope (C, N) 6-source model. Examination of carbon and nitrogen isotope plots indicated that some sources could be combined or eliminated. Spartina species (S. alterniflora and $S$. spartinae) were combined into 1 category, hereafter referred to as ' $\mathrm{C}_{4}$ plants', and Borrichia fruteccens and Iva frutescens were combined into a group, hereafter referred to as ' $\mathrm{C}_{3}$ plants'. The other potential sources were Ruppia maritima, phytomicrobenthos/SOM, filamentous algae and phytoplankton/SPM for a total of 6 potential sources. Nitrogen values of consumers were corrected prior to inclusion in Isosouce using 3.35 as the trophic fractionation value. Solutions that satisfied isotopic mass balance were examined in $1 \%$ increments within a tolerance of $0.01 \delta$ units. The mean and range of each source contribution is reported, because each individual solution satisfies mass balance and the mean alone may not represent the true contribution. To examine the potential influence of bias in the constant used for nitrogen trophic fractionation, the data set was analyzed with Isosource a second time using carbon data only, and results were compared.

Trophic level from isotopic data: Nitrogen isotopic distributions have been shown to be accurate indicators of trophic level in aquatic systems, where ${ }^{15} \mathrm{~N}$ enrichment increases predictably with trophic level of 
consumers (Vander Zanden \& Rasmussen 1999, Post 2002). The heavier ${ }^{15} \mathrm{~N}$ accumulates in consumers as nitrogen moves up the food web, and as a result top consumers tend to have higher values for $\delta^{15} \mathrm{~N}$ than consumers near the base of the web. Trophic levels of consumers were calculated following the method described in Jepsen \& Winemiller (2002). The formula used for the calculations of trophic level (TL) for a species was:

$$
\mathrm{TL}=\left[\left(\delta^{15} \mathrm{~N}_{\text {consumer }}-\delta^{15} \mathrm{~N}_{\text {reference }}\right) / 3.3\right]+1
$$

where $\delta^{15} \mathrm{~N}_{\text {reference }}$ (5.6) was the mean of all plant, sediment, phytoplankton/SPM, phytomicrobenthos/SOM, macroalgae, and filamentous algae samples, and the denominator value (3.3) was an estimated mean trophic enrichment (fractionation) of $\delta^{15} \mathrm{~N}$ between consumers and their food sources. The 3.3 estimate was calculated using dietary data from the pinfish Lagodon rhomboides. This common fish consumes comparable volumetric proportions of widgeon grass, vegetative detritus, filamentous algae, and amphipods. The mean nitrogen isotopic signature of these food items (5.66) was subtracted from the mean $\delta^{15} \mathrm{~N}$ value of pinfish (9.01). The resulting estimate of 3.3 agrees well with trophic fractionation values from laboratory studies of animal taxa fed high protein diets (DeNiro \& Epstein 1981, Minagawa \& Wada 1984, Vander Zanden \& Ramussen 1996, McCutchan et al. 2003).

Stomach contents: Trophic levels of fishes and macrocrustaceans were calculated for each season using the formula presented in Adams et al. (1983):

$$
\mathrm{TL}_{i}=1.0+\sum_{j=1}^{\mathrm{n}} \mathrm{TL}_{j}\left(p_{i j}\right)
$$

where $\mathrm{TL}_{i}$ is the trophic level of consumer species $i_{i} \mathrm{TL}_{j}$ is the trophic level of prey species $j$, and $p_{i j}$ is the fraction of the consumed food (volume) of species $i$ consisting of prey species $j$. Primary producers were coded as $\mathrm{TL}=1.0$ and primary consumers (feeding on only plant material) were $\mathrm{TL}=2.0$; therefore, a consumer eating exactly half plant and half herbivore tissue would have a TL of 2.5 .

\section{RESULTS}

\section{Relative abundance of macrofaunal taxa}

Nine taxa comprised $98.2 \%$ of the individual invertebrates obtained in twice monthly sediment core samples (Table 1). Amphipods and oligochaete worms comprised over $60 \%$ of the total. Grass shrimp strongly dominated the invertebrate sample from seine surveys, which more effectively captured larger invertebrates (Table 1). Forty-three fish species were captured in
Table 1. Abundances (density per unit effort) and percent relative abundances of macrofauna collected by 3 survey

\begin{tabular}{|c|c|c|}
\hline Species & \multicolumn{2}{|c|}{ Abundance \% } \\
\hline Sediment core samples & \multicolumn{2}{|l|}{ no. $\mathbf{m}^{-2}$} \\
\hline Amphipods & 1090.7 & 33.4 \\
\hline Oligochaete worms & 972.2 & 29.8 \\
\hline Gastropod mollusks & 349.1 & 10.7 \\
\hline Chironomid larvae & 279.2 & 8.6 \\
\hline Mysid shrimp & 178.7 & 5.5 \\
\hline Grass shrimp Palaemonetes pugio & 158.3 & 4.9 \\
\hline Polychaete worms & 133.3 & 4.1 \\
\hline Bivalve mollusks & 22.2 & 0.7 \\
\hline Crabs Callinectes spp. & 14.8 & 0.5 \\
\hline Additional 22 taxa & 62.1 & 1.8 \\
\hline Seine samples & \multicolumn{2}{|c|}{ no. $100 \mathrm{~m}^{-1}$} \\
\hline \multicolumn{3}{|l|}{ Invertebrates } \\
\hline Grass shrimp Palaemonetes pugio 2 & 2100.2 & 89.6 \\
\hline Brown shrimp Farfantepenaeus aztecus & 118.1 & 5.0 \\
\hline White shrimp Litopenaeus setiferus & 91.3 & 3.9 \\
\hline Blue crab Callinectes sapidus & 24.3 & 1.0 \\
\hline Additional 5 invertebrate taxa & 9.6 & 0.5 \\
\hline \multicolumn{3}{|l|}{ Fishes } \\
\hline Gulf menhaden Brevoortia patronus & 635.7 & 42.6 \\
\hline Bay anchovy Anchoa mitchilli & 546.7 & 36.6 \\
\hline Atlantic croaker Micropogonias undulatus & $s \quad 90.3$ & 6.0 \\
\hline Spot Leiostomus xanthurus & 41.3 & 2.8 \\
\hline Inland silverside Menidia beryllina & 36.4 & 2.4 \\
\hline Striped mullet Mugil cephalus & 28.1 & 1.9 \\
\hline Pinfish Lagodon rhomboides & 27.8 & 1.9 \\
\hline Tidewater silverside Menidia peninsulae & 25.5 & 1.7 \\
\hline Rainwater killifish Lucania parva & 11.5 & 0.8 \\
\hline $\begin{array}{l}\text { Sheepshead minnow } \\
\text { Cyprinodon variegatus }\end{array}$ & 11.3 & 0.8 \\
\hline Naked goby Gobiosoma bosc & 8.1 & 0.5 \\
\hline Silver croaker Bairdiella chrysoura & 7.5 & 0.5 \\
\hline Additional 31 taxa & 51.7 & 3.4 \\
\hline Gillnet samples & \multicolumn{2}{|c|}{ no. $h^{-1}$} \\
\hline Striped mullet Mugil cephalus & 0.73 & 34.0 \\
\hline Gizzard shad Dorosoma cepedianum & 0.52 & 24.3 \\
\hline Red drum Sciaenops ocellatus & 0.25 & 11.8 \\
\hline Hardhead catfish Arius felis & 0.21 & 9.8 \\
\hline Black drum Pogonias cromis & 0.12 & 5.7 \\
\hline Southern flounder Paralichthys lethostigma & 0.09 & 4.0 \\
\hline Alligator gar Atractosteus spatula & 0.08 & 3.8 \\
\hline Spotted seatrout Cynoscion nebulosus & 0.04 & 1.7 \\
\hline Ladyfish Elops saurus & 0.02 & 0.9 \\
\hline Atlantic croaker Micropogonias undulatus & 0.02 & 0.8 \\
\hline Spotted gar Lepisosteus oculatus & 0.02 & 0.7 \\
\hline Spot Leiostomus xanthurus & 0.01 & 0.5 \\
\hline Atlantic stingray Dasyatis sabina & 0.01 & 0.5 \\
\hline Additional 7 taxa & 0.03 & 1.0 \\
\hline
\end{tabular}
methods at Mad Island Marsh over the 18 mo survey

seine surveys; 2 fish species, bay anchovy and Gulf menhaden, were numerically dominant in seine samples (together comprising $79 \%$ of all individuals captured; Table 1). Gillnet surveys that targeted larger fishes yielded 20 fish species; 2 detritivorous species, striped mullet and gizzard shad Dorosoma cepedianum, comprised more than half the individuals captured in gillnets (Table 1). 


\section{Sources of carbon for consumers: evidence from carbon isotope ratios}

Plant samples from MIM revealed differences in mean $\delta^{13} \mathrm{C}$ values between most of the major categories and relatively low variation within categories (Table 2), but it should be noted that variation was not examined. $\mathrm{C}_{4}$ macrophytes (Cynodon dactylon, Spartina alterniflora, S. spartinae) were relatively enriched in ${ }^{13} \mathrm{C}$ and had mean $\delta^{13} \mathrm{C}$ values between -12.7 and $-13.4 \%$, a range that corresponds to values reported for other $\mathrm{C}_{4}$ plants (Fry et al. 1977, Peterson \& Fry 1987). Ruppia maritima, an aquatic $\mathrm{C}_{3}$ plant, also had a low $\delta^{13} \mathrm{C}$ value $(-14.6 \pm 0.4)$, perhaps due to utilization of bicarbonate and internal dissolved inorganic $\mathrm{C}$ recycling. $\mathrm{C}_{3}$ terrestrial plants from upland areas of the marsh were more depleted in ${ }^{13} \mathrm{C}$. Borrichia fruteccens (bushy seaside tansy) and Iva frustesscens (marsh elder) had mean $\delta^{13} \mathrm{C}$ values of -25.4 and $-28.5 \%$, respectively, within the range reported by Smith \& Epstein (1971) for terrestrial $\mathrm{C}_{3}$ plants (-23 to $-30 \%$ ). Values for phytoplankton/SPM, filamentous algae, phytomicrobenthos/SOM, and macroalgae (ranging from -13 to $-22 \%$, Table 2) overlapped with both $\mathrm{C}_{3}$ and $\mathrm{C}_{4}$ terrestrial plants, a finding consistent with those from other estuarine systems (Peterson \& Fry 1987).

With few exceptions, invertebrates at MIM had $\delta^{13} \mathrm{C}$ values that were intermediate relative to the overall range observed for plants (Table 2, Fig. 1). Amphipods and grass shrimp had heaviest mean $\delta^{13} \mathrm{C}$ values, indicating that these taxa assimilate carbon derived mostly from $\mathrm{C}_{4}$ plants and/or Ruppia maritima. Isosource model estimates of material assimilated from 6 primary production sources revealed that $\mathrm{C}_{4}$ grasses were more important than $R$. maritima for supporting biomass of amphipods and grass shrimp (Table 3). Nearly all the other invertebrates had $\delta^{13} \mathrm{C}$ values ranging from -18.4 to $-22.8 \%$, a range broadly overlapping the $\delta^{13} \mathrm{C}$ values of algae/particulate organic matter groups in this system. Isosource estimates with both $\delta^{13} \mathrm{C}$ and $\delta^{15} \mathrm{~N}$ data indicated that filamentous algae and $\mathrm{C}_{3}$ terrestrial plants were probably the most important primary production sources supporting biomass of these invertebrates (Table 3), however minor contributions from $\mathrm{C}_{4}$ grasses, $R$. maritima, phytoplankton, and phytomicrobenthos were also possible.

Table 2. $\delta^{13} \mathrm{C}$ and $\delta^{15} \mathrm{~N}$ values of samples from study area $(x \pm \mathrm{SD}, \mathrm{n}=$ sample size). Codes are used in Fig. 1

\begin{tabular}{|c|c|c|c|c|}
\hline & Code & $\mathrm{n}$ & $\delta^{13} \mathrm{C}$ & $\delta^{15} \mathrm{~N}$ \\
\hline Filamentous algae & FIL & 4 & $-18.7 \pm 0.3$ & $6.3 \pm 0.1$ \\
\hline Phytomicrobenthos/SOM & PMB & 3 & $-18.4 \pm 2.3$ & $2.8 \pm 0.2$ \\
\hline Phytoplankton/SPM & PHY & 3 & $-20.0 \pm 2.8$ & $3.5 \pm 0.7$ \\
\hline \multicolumn{5}{|l|}{ Macroalgae } \\
\hline Chara sp. & CHA & 3 & $-16.2 \pm 0.1$ & $0.7 \pm 0.3$ \\
\hline Ulva sp. & MALG & 3 & $-20.2 \pm 0.2$ & $6.5 \pm 0.4$ \\
\hline \multicolumn{5}{|l|}{ Vascular salt marsh plants } \\
\hline Spartina alterniflora & SAL & 5 & $-12.8 \pm 0.1$ & $6.5 \pm 4.1$ \\
\hline Spartina spartinae & SPA & 3 & $-13.0 \pm 0.1$ & $5.4 \pm 0.3$ \\
\hline Ruppia maritima & RM & 4 & $-14.6 \pm 0.4$ & $4.6 \pm 0.4$ \\
\hline Cynodon dactylon & CYD & 2 & $-13.4 \pm 0.2$ & $4.7 \pm 0.1$ \\
\hline Borrichia fruteccens & $\mathrm{BO}$ & 3 & $-28.5 \pm 0.2$ & $6.4 \pm 1.2$ \\
\hline Iva frutescens & IVA & 2 & $-25.4 \pm 0.4$ & $5.0 \pm 0.2$ \\
\hline $\begin{array}{l}\text { Mud and fine particulate } \\
\text { organic matter }\end{array}$ & FPM & 2 & $-13.5 \pm 0.2$ & $8.7 \pm 1.0$ \\
\hline Coarse vegetative detritus & VEGD & 6 & $-14.4 \pm 0.9$ & $5.3 \pm 0.4$ \\
\hline Zooplankton & $\mathrm{ZOO}$ & 1 & -20.7 & 7.2 \\
\hline \multicolumn{5}{|l|}{ Invertebrates } \\
\hline Amphipoda & AMP & 2 & $-14.8 \pm 0.3$ & $6.4 \pm 0.1$ \\
\hline Grass shrimp Palaemonetes pugio & PG & 4 & $-15.2 \pm 0.3$ & $11.9 \pm 0.3$ \\
\hline $\begin{array}{l}\text { Brown shrimp } \\
\quad \text { Farfantepenaeus aztecus }\end{array}$ & FA & 9 & $-18.4 \pm 0.6$ & $8.0 \pm 0.5$ \\
\hline White shrimp Litopenaeus setiferus & LSE & 5 & $-20.6 \pm 0.2$ & $10.1 \pm 3.3$ \\
\hline Blue crab Callinectes sapidus & $\mathrm{CS}$ & 7 & $-19.3 \pm 1.4$ & $11.1 \pm 2.3$ \\
\hline Mussel unidentified bivalve & MUS & 1 & -22.2 & 8.0 \\
\hline Gastropod Littorina sp. & GAS & 1 & -8.1 & 5.8 \\
\hline Oyster & OYS & 4 & $-22.76 \pm 0.3$ & $10.2 \pm 1.3$ \\
\hline \multicolumn{5}{|l|}{ Fishes } \\
\hline Bay anchovy Anchoa mitchilli & $\mathrm{AM}$ & 9 & $-20.3 \pm 0.4$ & $12.8 \pm 0.8$ \\
\hline Hardhead catfish Arius felis & $\mathrm{AF}$ & 6 & $-19.0 \pm 1.3$ & $13.7 \pm 1.0$ \\
\hline Alligator gar Atractosteus spatula & AS & 2 & $-18.1 \pm 1.2$ & $13.2 \pm 0.3$ \\
\hline Silver perch Bairdiella chrysoura & $\mathrm{BC}$ & 4 & $-19.3 \pm 0.5$ & $14.7 \pm 2.0$ \\
\hline Gulf menhaden Brevoortia patronus & $\mathrm{BP}$ & 4 & $-20.5 \pm 1.6$ & $14.5 \pm 0.4$ \\
\hline $\begin{array}{l}\text { Spotted seatrout } \\
\text { Cynoscion nebulosus }\end{array}$ & $\mathrm{CN}$ & 8 & $-19.4 \pm 0.6$ & $13.5 \pm 2.2$ \\
\hline $\begin{array}{l}\text { Sheepshead minnow } \\
\text { Cyprinodon variegatus }\end{array}$ & $\mathrm{CV}$ & 1 & -14.4 & 9.6 \\
\hline Gizzard shad Dorosoma cepedianum & $\mathrm{DC}$ & 8 & $-19.9 \pm 0.8$ & $12.5 \pm 0.8$ \\
\hline Ladyfish Elops saurus & ES & 4 & $-16.5 \pm 0.5$ & $9.3 \pm 1.5$ \\
\hline Gulf killifish Fundulus grandis & FG & 4 & $-15.6 \pm 0.9$ & $9.2 \pm 1.2$ \\
\hline Naked goby Gobiosoma bosc & GB & 2 & $-17.2 \pm 0.3$ & $9.1 \pm 0.5$ \\
\hline Pinfish Lagodon rhomboides & LR & 12 & $-16.9 \pm 1.4$ & $9.0 \pm 1.7$ \\
\hline Spot Leiostomus xanthurus & LX & 2 & $-13.5 \pm 0.1$ & $13.3 \pm 2.0$ \\
\hline Spotted gar Lepisosteus oculatus & LO & 1 & -17.1 & 12.4 \\
\hline Rainwater killifish Lucania parva & LP & 1 & -17.3 & 9.5 \\
\hline Inland silverside Menidia beryllina & $\mathrm{MB}$ & 6 & $-19.2 \pm 0.5$ & $11.1 \pm 0.4$ \\
\hline Striped mullet Mugil cephalus & $\mathrm{MC}$ & 8 & $-17.4 \pm 0.9$ & $10.4 \pm 1.8$ \\
\hline $\begin{array}{l}\text { Southern flounder } \\
\text { Paralichthys lethostigma }\end{array}$ & PLE & 2 & $-18.5 \pm 0.1$ & $13.2 \pm 1.7$ \\
\hline Black drum Pogonias cromis & $\mathrm{PC}$ & 8 & $-18.8 \pm 1.1$ & $12.9 \pm 1.1$ \\
\hline Red drum Sciaenops ocellatus & $\mathrm{SO}$ & 13 & $-17.9 \pm 0.6$ & $13.3 \pm 0.9$ \\
\hline Gulf pipefish Syngnathus scovelli & SS & 1 & -18.7 & 10.8 \\
\hline
\end{tabular}




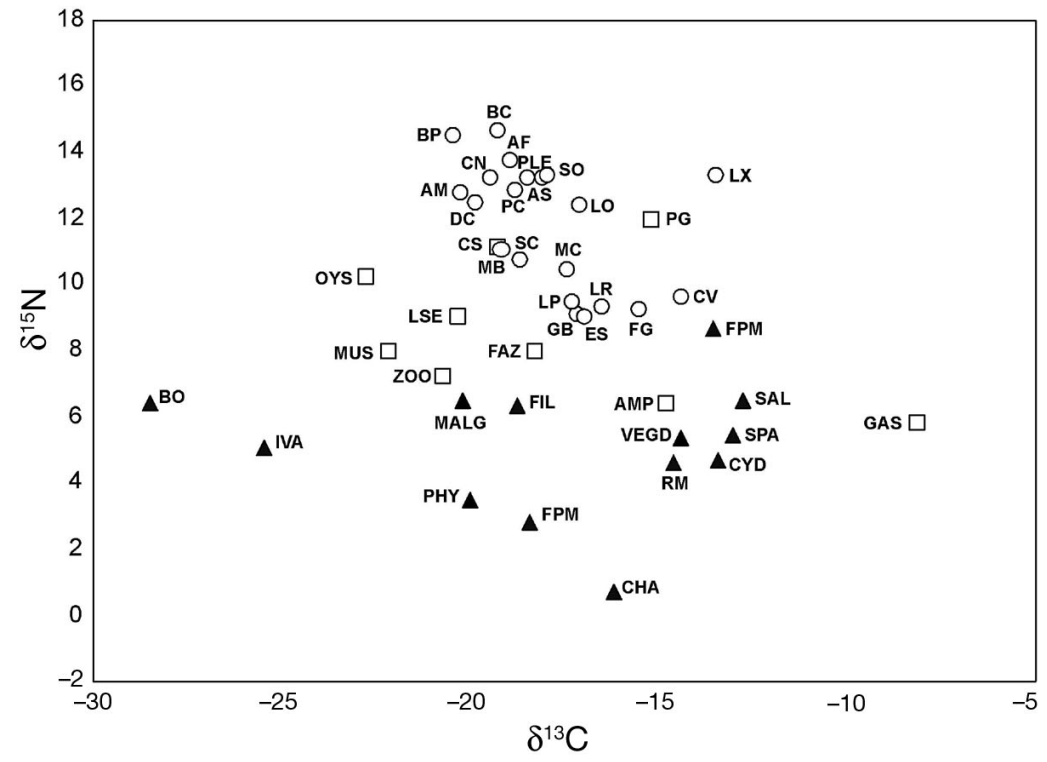

Fig. 1. $\delta^{15} \mathrm{~N}$ and $\delta^{13} \mathrm{C}$ values for major food web elements in Mad Island Marsh. $\boldsymbol{\Delta}$ : primary production sources; $\square$ : invertebrates; O: fishes; codes as in Table 2. Potential assimilation of carbon sources by consumers is indicated by degree of alignment among taxa relative to $x$-axis values, trophic level by relative position on $y$-axis
Fishes at MIM had $\delta^{13} \mathrm{C}$ values (Table 2, Fig. 1) that indicated assimilation of carbon from a mixture of primary production sources. According to the Isosource model estimates, large fractions of material originating from emergent $\mathrm{C}_{4}$ macrophytes were probably assimilated into tissues of several small fishes that inhabit densely vegetated, marginal areas of the marsh, e.g. spot and Gulf killifish (Table 3). Isosource results indicated that most other fish species assimilated variable mixtures of material derived from $\mathrm{C}_{4}$ and $\mathrm{C}_{3}$ plants and filamentous algae, with Ruppia maritima, phytomicrobenthos/SOM and phytoplankton/SPM estimated to be of minor importance (Table 3). According to these model estimates, $\mathrm{C}_{4}$ grasses were probably the dominant source supporting biomass of 7 fish species, $C_{3}$ plants were the dominant source for Gulf menhaden only, filamentous algae

Table 3. Two-element, multiple-source model estimates of mean percent of carbon in consumer tissues derived from 5 potential basal sources (values in parentheses are ranges of possible percentages)

\begin{tabular}{|c|c|c|c|c|c|c|}
\hline & $\begin{array}{c}\mathrm{C}_{4} \\
\text { plants }\end{array}$ & $\begin{array}{c}\mathrm{C}_{3} \\
\text { plants }\end{array}$ & $\begin{array}{l}\text { Ruppia } \\
\text { maritima }\end{array}$ & $\begin{array}{l}\text { Phytomicro- } \\
\text { benthos/SOM }\end{array}$ & $\begin{array}{c}\text { Phyto- } \\
\text { plankton/SPM }\end{array}$ & $\begin{array}{c}\text { Filamentous } \\
\text { algae }\end{array}$ \\
\hline \multicolumn{7}{|l|}{ Fishes } \\
\hline Leiostomus xanthurus & $76(72-80)$ & $0(0-0)$ & $19(14-26)$ & $3(0-6)$ & $1(0-2)$ & $<1(0-1)$ \\
\hline Cyprinodon variegatus & $71(52-82)$ & $3(0-7)$ & $11(0-31)$ & $4(0-13)$ & $5(0-16)$ & $7(0-17)$ \\
\hline Fundulus grandis & $56(29-74)$ & $6(0-16)$ & $11(0-36)$ & $5(0-15)$ & $5(0-18)$ & $17(0-35)$ \\
\hline Elops saurus & $43(12-67)$ & $9(0-23)$ & $12(0-38)$ & $5(0-17)$ & $6(0-20)$ & $26(0-50)$ \\
\hline Lagodon rhomboides & $37(1-64)$ & $10(0-26)$ & $12(0-42)$ & $5(0-17)$ & $6(0-22)$ & $30(0-57)$ \\
\hline Gobiosoma bosc & $35(0-63)$ & $11(0-27)$ & $11(0-40)$ & $5(0-17)$ & $6(0-21)$ & $33(0-61)$ \\
\hline Lepisosteus oculatus & $33(0-64)$ & $10(0-27)$ & $14(0-44)$ & $6(0-21)$ & $7(0-24)$ & $31(0-56)$ \\
\hline Lucania parva & $33(0-62)$ & $11(0-28)$ & $12(0-40)$ & $5(0-18)$ & $6(0-21)$ & $34(0-62)$ \\
\hline Mugil cephalus & $31(0-61)$ & $11(0-29)$ & $12(0-38)$ & $5(0-18)$ & $6(0-22)$ & $35(0-63)$ \\
\hline Sciaenops ocellatus & $25(0-58)$ & $13(0-33)$ & $11(0-37)$ & $5(0-18)$ & $6(0-22)$ & $40(0-71)$ \\
\hline Atractosteus spatula & $24(0-57)$ & $14(0-34)$ & $11(0-37)$ & $5(0-18)$ & $7(0-23)$ & $40(0-72)$ \\
\hline Paralichthys lethostigma & $21(0-54)$ & $16(0-37)$ & $10(0-35)$ & $5(0-19)$ & $7(0-23)$ & $42(0-78)$ \\
\hline Syngnathus scovelli & $20(0-53)$ & $18(0-39)$ & $9(0-33)$ & $5(0-19)$ & $7(0-24)$ & $42(0-78)$ \\
\hline Arius felis & $19(0-59)$ & $20(0-40)$ & $9(0-33)$ & $5(0-19)$ & $7(0-24)$ & $41(0-77)$ \\
\hline Pogonias cromis & $19(0-52)$ & $19(0-39)$ & $9(0-34)$ & $5(0-20)$ & $7(0-24)$ & $41(0-78)$ \\
\hline Menidia beryllina & $18(0-49)$ & $22(2-42)$ & $9(0-33)$ & $5(0-19)$ & $7(0-25)$ & $39(0-74)$ \\
\hline Bairdiella chrysoura & $18(0-49)$ & $23(5-43)$ & $9(0-31)$ & $5(0-18)$ & $7(0-22)$ & $38(0-73)$ \\
\hline Cynoscion nebulosus & $18(0-48)$ & $25(5-44)$ & $9(0-33)$ & $5(0-18)$ & $7(0-25)$ & $37(0-71)$ \\
\hline Dorosoma cepedianum & $17(0-44)$ & $29(11-47)$ & $8(0-30)$ & $5(0-17)$ & $6(0-22)$ & $35(0-68)$ \\
\hline Anchoa mitchilli & $16(0-42)$ & $33(16-50)$ & $8(0-29)$ & $5(0-17)$ & $6(0-22)$ & $33(0-63)$ \\
\hline Brevoortia patronus & $15(0-41)$ & $36(19-51)$ & $8(0-29)$ & $5(0-16)$ & $6(0-20)$ & $31(0-31)$ \\
\hline \multicolumn{7}{|l|}{ Invertebrates } \\
\hline Amphipoda & $66(45-79)$ & $4(0-10)$ & $10(0-31)$ & $4(0-14)$ & $5(0-16)$ & $11(0-24)$ \\
\hline Palaemonetes pugio & $61(35-76)$ & $5(0-13)$ & $11(0-35)$ & $4(0-15)$ & $5(0-18)$ & $14(0-30)$ \\
\hline Farfantepenaeus aztecus & $22(0-56)$ & $15(0-36)$ & $10(0-34)$ & $5(0-19)$ & $6(0-23)$ & $42(0-75)$ \\
\hline Litopenaeus setiferus & $16(0-42)$ & $34(17-50)$ & $8(0-30)$ & $5(0-17)$ & $6(0-21)$ & $32(0-62)$ \\
\hline Callinectes sapidus & $15(0-54)$ & $21(4-44)$ & $9(0-33)$ & $5(0-18)$ & $6(0-25)$ & $44(0-72)$ \\
\hline Zooplankton & $15(0-39)$ & $38(22-53)$ & $7(0-25)$ & $4(0-16)$ & $6(0-20)$ & $31(0-59)$ \\
\hline Mussel & $11(0-30)$ & $53(40-64)$ & $6(0-22)$ & $3(0-12)$ & $5(0-16)$ & $22(0-44)$ \\
\hline Oyster & $10(0-25)$ & $58(47-68)$ & $6(0-22)$ & $4(0-12)$ & $5(0-16)$ & $18(0-38)$ \\
\hline
\end{tabular}


was the dominant source for 12 species, and $\mathrm{C}_{3}$ plants and filamentous algae were equally important for bay anchovy. Given that algae isotopic signatures often show significant temporal and spatial variation in estuaries (e.g. Creach et al. 1997, Currin et al. 2003, Cook et al. 2004), these model estimates of primary production source contributions to consumer tissues should be considered tentative pending additional sampling of algae during different seasons.

\section{Sources of carbon for consumers: evidence from dietary analysis}

The total volume of food items removed from 6452 fish and crustacean stomachs was $3.70 \mathrm{l}$. Among 66 prey categories identified, the major food items consumed by fishes and invertebrates were (as percentage of total volume extracted) detritus (32\%), striped mullet $(19 \%)$, blue crab $(15 \%)$, snails $(6 \%)$, unicellular green algae $(4 \%)$, diatoms $(3 \%)$, pinfish $(2 \%)$, white shrimp $(1 \%)$, brown shrimp $(1 \%)$, widgeon grass $(1 \%)$, black drum $(1 \%)$, and gar $(1 \%)$. All other food categories had percentage volumes less than $1 \%$. Nonetheless, for some species of small or rare consumer taxa, several of these food resources comprised major elements of trophic pathways supporting their biomass. For example, bivalve mollusks comprised $51 \%$ of the diet of the black drum by volume, and calanoid copepods comprised $14 \%$ of the bay anchovy diet.

We examined the diets of the 5 most abundant primary consumers (in terms of biomass) based on the complete annual sample of stomach contents (Table 4). Detritus was by far the dominant food resource consumed by grass shrimp, striped mullet, Gulf menhaden, and gizzard shad. Gulf menhaden in the marsh were juveniles that consumed a large fraction of centric diatoms in addition to a major detrital component. Pinfish consumed mostly Ruppia maritima (70\% volume) and invertebrates (18.5\%).

Diets of the abundant large carnivorous fishes were dominated by detritivorous fishes and crustaceans that fed mainly on detritus and/or phytomicrobenthos. For example, striped mullet was an important prey for alligator gar ( $83 \%$ of diet by volume), speckled seatrout $(70 \%)$, spotted gar $(62 \%)$, and red drum $(10 \%)$. Pinfish were consumed by southern flounder $(41.4 \%)$, spotted gar $(30.4 \%)$, hardhead catfish $(10 \%)$, and speckled seatrout $(6.2 \%)$. Blue crabs were consumed by red drum $(70 \%)$ and hardhead catfish $(24 \%)$. Detritivorous juvenile Gulf menhaden were consumed by southern flounder $(29 \%)$, speckled seatrout $(4 \%)$, and red drum $(1 \%)$. Thus, our analysis of consumer diets indicates that detritus is the dominant source of plant material supporting most consumers in the MIM food web. However, the specific source of fine particulate organic matter removed from stomachs is nearly impossible to determine by microscopic examination.

\section{Vertical trophic structure: evidence from nitrogen isotope ratios}

Our analysis of stable nitrogen isotope ratios of the most abundant consumer taxa indicates approximately 4 trophic levels in this aquatic ecosystem (Fig. 2). Four invertebrates (including zooplankton in the aggregate) and 8 fish species approximated the second trophic level (TL) of primary consumers (TL values from 1.24 to 2.66). Three consumer taxa (amphipods, zooplankton, and white shrimp) actually had values less than 2.0. Error in the zooplankton estimate could have been associated with contamination of samples with particu-

Table 4. Volumetric proportion of detritus, algae, and aquatic macrophytes in diets of common primary consumers (grass shrimp, striped mullet, Gulf menhaden, gizzard shad, and pinfish) at Mad Island Marsh, and the total volume (ml) of each diet category taken from stomachs of the entire consumer sample

\begin{tabular}{|c|c|c|c|c|c|c|}
\hline & $\begin{array}{c}\text { Palaemonetes } \\
\text { pugio }\end{array}$ & $\begin{array}{l}\text { Mugil } \\
\text { cephalus }\end{array}$ & $\begin{array}{c}\text { Brevoortia } \\
\text { patronus }\end{array}$ & $\begin{array}{c}\text { Dorosoma } \\
\text { cepedianum }\end{array}$ & $\begin{array}{c}\text { Lagodon } \\
\text { rhomboides }\end{array}$ & Total \\
\hline Centric diatoms & 0.017 & 0.003 & 0.138 & 0.001 & 0.005 & 6.64 \\
\hline Pennate diatoms & 0.020 & 0.007 & 0.025 & 0.004 & 0.002 & 7.70 \\
\hline Dinoflagellates (Noctiluca spp.) & & 0.001 & 0.002 & & & 0.79 \\
\hline Unicellular green algae & & 0.093 & 0.002 & 0.042 & & 75.40 \\
\hline Filamentous green algae & 0.049 & 0.020 & 0.013 & 0.009 & 0.047 & 20.05 \\
\hline Oscillatoria spp. & 0.001 & 0.015 & & 0.003 & 0.006 & 10.21 \\
\hline Other cyanobacteria & 0.002 & 0.009 & 0.002 & 0.005 & & 8.42 \\
\hline Chara spp. & & & & & 0.026 & 0.44 \\
\hline Ruppia maritima & 0.005 & 0.007 & & 0.003 & 0.698 & 27.85 \\
\hline Other aquatic macrophytes & & & & & 0.001 & 1.26 \\
\hline Detritus & 0.837 & 0.658 & 0.789 & 0.783 & 0.030 & 62.53 \\
\hline
\end{tabular}




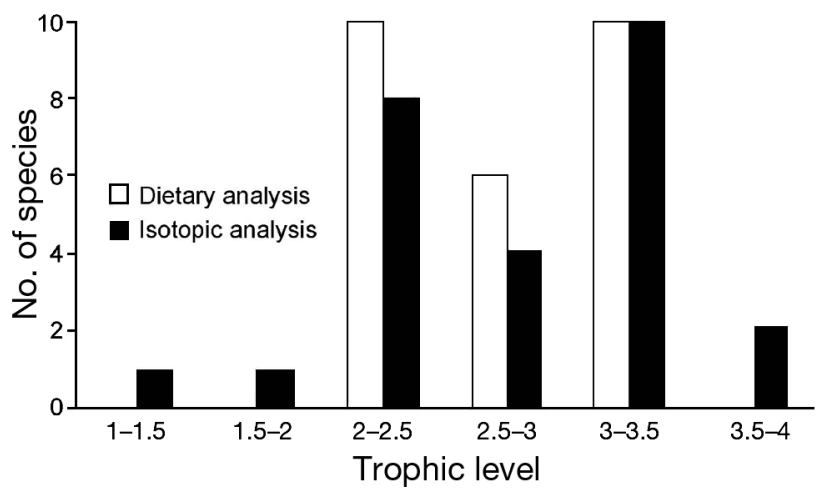

Fig. 2. Frequency distributions of trophic levels of Mad Island Marsh taxa based on $\delta^{15} \mathrm{~N}$ data (filled bars) and volumetric dietary data (open bars)

late matter of plant origin. Another source of error in estimating trophic levels could have been variation in $\delta^{15} \mathrm{~N}$ among presumptive source taxa (TL 1) consumed in variable proportions by these taxa. The third trophic level (TL 2.6 to 3.4) contained 11 fish and 2 invertebrates species. The largest piscivorous fishes were at the third trophic level: spotted gar $=3.0$, alligator gar $=$ 3.3 , southern flounder $=3.3$, spotted seatrout $=3.3$, red drum = 3.3). The species that approached a fourth trophic level were fishes that consumed variable amounts of zooplankton: silver perch (TL $=3.7$ ) and juvenile gulf menhaden $(\mathrm{TL}=3.65)$.

\section{Comparison of vertical trophic structure derived from dietary analyses}

Based on the same set of abundant consumer taxa (except for the amphipod, for which we present isotopic data but had no dietary data), we compared trophic level estimates calculated using dietary data with estimates calculated from stable isotope data. With 3 exceptions, the 2 methods yielded fairly concordant results (Fig. 3). The 3 taxa with highly discordant trophic levels based on the 2 methods were Gulf menhaden, gizzard shad, and grass shrimp. All 3 of these species had much higher trophic levels calculated from the stable isotope analysis compared to estimates based on stomach contents analysis. When we removed these organisms from the statistical analysis, the coefficient of determination for the linear regression increased from 0.185 to 0.60 . All 3 of these outlying species consumed large volumetric proportion of detritus (Table 4), but also minor and variable proportions of algae and invertebrates, such as protozoans, nematodes, microcrustaceans, and mollusks. In contrast to these detritivores, the ladyfish, a carnivore, had a lower estimate of trophic level based on the isotopic data (diet $\mathrm{TL}=3.07$, isotopic $\mathrm{TL}=2.12$ ).

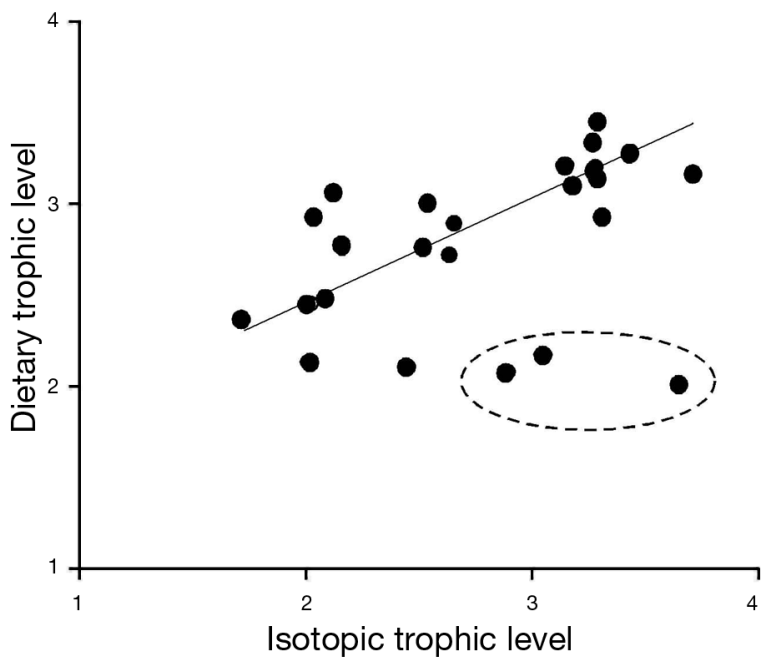

Fig. 3. Comparison of trophic level values for Mad Island Marsh consumer species derived from $\delta^{15} \mathrm{~N}$ vs. volumetric dietary data. Three outlying species (inside oval) were Brevoortia patronus, Palaemonetes pugio, and Dorosoma cepedianum $\left(\mathrm{r}^{2}\right.$ for full data set $=0.18, \mathrm{p}=0.04 ; \mathrm{r}^{2}$ for data set without 3 outliers $=0.60, \mathrm{p}<0.0001$ )

According to the isotopic method, the amphipod had a trophic level of 1.2 and the brown shrimp had TL = 1.7. These estimates are obviously biased, given that the lowest possible consumer level is 2.0. The lowest trophic levels based on the dietary method were for the sheepshead minnow (TL $=2.0)$, Gulf menhaden $(\mathrm{TL}=$ 2.0), and grass shrimp (2.1). The species with the highest trophic levels according to dietary analysis were red drum $(\mathrm{TL}=3.4)$, southern flounder $(\mathrm{TL}=3.3$ ), and spotted seatrout $(\mathrm{TL}=3.3)$. These species had essentially the same trophic levels according to the isotopic methodology (3.3 in each case).

Several abundant fish species were not encountered in any predator stomachs, even though many of these species are known to be exploited by a variety of piscivorous fishes based on other studies. For example no gizzard shad were recovered from stomachs during our study, yet this species is exploited by gars in freshwater habitats of Texas (K. O. Winemiller unpubl. data) and other regions (Garcia et al. 2001).

\section{DISCUSSION}

A previous analysis of seasonal variation in food web structure at MIM that was based entirely on dietary information (Akin \& Winemiller 2006) revealed low temporal variation in most general food web properties, even though community composition and population size structure of certain species changed. Proportions of top (taxa with no observed predators), 
intermediate (species observed as both predator and prey), and basal (primary producers) taxa did not vary significantly between seasons. The summer food web had a few more taxa and trophic links than the winter web, and the volume of consumed material (estimated from collective stomach volume of the species assemblage) and mean trophic level were greater during summer. In the current study, dietary data collected over 18 mo were combined into an annual food web for comparison with isotopic data from consumer tissues collected during August. Muscle tissue should reflect the elemental composition of material assimilated during the preceding several weeks to months, with the interval depending on factors such as body size and trophic position (O'Reilly et al. 2002). Thus, a potential source of bias in our analysis would be mismatches in temporal resolution of dietary and isotopic data sets. Another potential source of bias is spatial variation; for example, migratory species might reflect material assimilated from coastal marine waters rather than local sources (Herzka 2005). Most of the marine immigrants at MIM were small juveniles of estuarinedependent marine species, such as Gulf menhaden and various sciaenids, assumed to have fed and grown almost entirely in situ. Nonetheless, some of the large fishes could have entered the system following periods of residence in other areas, such as the coastal Gulf of Mexico, Matagorda Bay, or freshwater creeks.

\section{Production sources and horizontal web structure}

Dietary analyses revealed consumption of large amounts of detritus, both vegetative (macrophyte tissues) and fine particulate, by species that were among the most abundant in the system, such as amphipods, striped mullet and gizzard shad. Stable isotope analysis indicated that these abundant detritivores probably assimilated carbon derived from variable mixtures of algal and macrophyte sources. Emergent cordgrasses dominate shallow marginal areas of the marsh throughout the year. Submerged widgeon grass is abundant in deeper areas during summer and fall, then dies back during winter. Detritus from widgeon grass is probably present in sediments throughout the year. Despite the fact that emergent cordgrasses dominate the standing plant biomass of the system, these plants are consumed directly by very few organisms. Periwinkle snails are significant grazers of cordgrasses (Silliman \& Zieman 2001), and our single periwinkle sample was highly ${ }^{13} \mathrm{C}$-enriched $\left(\delta^{13} \mathrm{C}=-8.1\right)$, probably reflecting consumption of cordgrass and perhaps contamination of our soft tissue sample with carbonate from shell fragments. In contrast to our analysis, Peterson \& Howarth's (1987) study of a Georgia saltmarsh found Littorina spp. $\left(\delta^{13} \mathrm{C}=-14.6\right)$ to have slightly lighter carbon signature than Spartina spp. (-12.9), and concluded that, like virtually all of the macrofauna investigated, periwinkles assimilate some fraction of carbon derived from both Spartina spp. and phytoplankton. Most Spartina spp. biomass is probably consumed as detritus. Studies have revealed little fractionation of carbon isotope ratios within dead Spartina spp. tissue after processing by bacteria $(<2 \%$ o) or within bacteria (1 to $2 \%$ ) growing on Spartina spp. detritus (Coffin et al. 1988, Currin et al. 1995, Boschker et al. 1999). Oysters and another bivalve mollusk had carbon isotope ratios that were $2 \%$ lighter than the lightest algal, particulate organic matter, and $\mathrm{C}_{3}$ macrophyte samples. Because we only collected phytoplankton (fine seston) samples from the upper and middle reaches of the system during the late summer, it is likely that these bivalves reflected ratios of phytoplankton with lower $\delta^{13} \mathrm{C}$ from the lower estuary or from other locations that were delivered on tides.

Previous stable isotope studies of saltmarsh food webs in North America have concluded that macrofauna are supported mostly by a combination of $\mathrm{C}_{4}$ marsh grasses, algae, and to a lesser extent terrestrial $\mathrm{C}_{3}$ plants, with the relative fraction of each depending on trophic niche, size class, season, and location (Haines \& Montague 1979, Peterson \& Howarth 1987 , Deegan \& Garritt 1997, Kwak \& Zedler 1997). Our Isosource results conform to this pattern, with some MIM consumers (e.g. amphipods, spot) assimilating greater fractions of carbon derived from macrophytes, and others (e.g. southern flounder, red drum) assimilating greater fractions of material derived from algae, filamentous algae in particular. To some extent, source material assimilated by consumers seemed to be associated with spatial distributions. Killifishes were estimated to assimilate large fractions of $\mathrm{C}_{4}$-derived material, and were also captured from shallow marginal waters, usually within or near dense stands of $\mathrm{C}_{4}$ marsh grasses. Amphipods were captured from organic-rich sediments and dense stands of vegetation throughout the marsh, and also reflected a large $\mathrm{C}_{4}$ macrophyte contribution. Midwater planktivorous fishes, such as Gulf menhaden, bay anchovy and inland silversides, were estimated to derive carbon mostly from filamentous algae and $\mathrm{C}_{3}$ plants, and were captured from more open and deeper regions of the marsh. Diatoms and filamentous algae grow on the surfaces of submerged living as well as dead macrophytes. Our isotopic samples were not sufficient to permit investigation of spatial patterns, and it would be interesting to examine variation associated with the longitudinal salinity gradient at this site (Akin et al. 2003). Previous studies of coastal systems have revealed greater spatial variation than temporal variation in most species (Deegan \& Garritt 
1997, Weinstein et al. 2000, Bouillon et al. 2002, Melville \& Connolly 2003).

Although isotopic analysis performed better than dietary analysis in revealing primary production sources supporting consumers, it could not reveal the detailed structure of predator-prey interactions at the species level. Stomach contents analysis directly identified many consumer-resource interactions, particularly for consumers at higher trophic levels. Yet dietary analysis probably overestimated the importance of many production sources consumed by detritivores, the dominant consumer trophic guild in terms of numerical abundance. Microscopic examination of stomach contents of most detritivores (fishes, crustaceans) revealed large fractions of fine amorphous organic material that were assumed to be derived from various fractions of macrophyte and algal biomass. This material was also assumed to contain heterotrophic bacteria. Additional laboratory methods, such as analysis of nucleic acids, lipids or pigments (Canuel et al. 1995, Cook et al. 2004), would be needed to identify the origin of this material. Another well-recognized problem with stomach contents analysis is that material has variable nutritional value for the consumer. Much of the coarse vegetative detritus consumed by fishes and crustaceans was probably refractory and not assimilated.

\section{Vertical web structure}

Estimates of vertical web structure (consumer trophic levels) by the 2 methods were largely concordant. The exceptions were zooplanktivorous and detritivorous fishes, which had higher trophic levels according to nitrogen isotope ratios compared with estimates from dietary data. It is likely that the isotope method more accurately indexed the number of trophic transfers than the dietary method, which depends on accurate dietary estimation for all elements of food chains leading to a consumer, and which assumes equal assimilation efficiencies for elements found in stomach contents. The isotope method placed juvenile Gulf menhaden near the top of the food web, whereas the dietary method placed it at the second trophic level. Menhaden stomachs contained mostly fine amorphous organic material that was assumed to derive from a mixture of algal and macrophyte sources. Only small amounts of invertebrates were recovered from stomachs. Isotopic analysis inferred that invertebrates actually were the primary nutritional resource for menhaden. Thus, juvenile menhaden could best be characterized as zooplanktivores positioned at Trophic Level 3, which is similar to the conclusion obtained from stable isotope studies of menhaden in a coastal marsh in Georgia (Peterson \& Howarth 1987). Gulf menhaden use coastal marshes as a nursery habitat, with larger juveniles moving offshore during the late summer. Gizzard shad and striped mullet also had higher trophic level estimates according to the isotopic method. These species feed on invertebrates as juveniles then become increasingly detritivorous as they grow. Stomach contents of these species consisted mostly of detritus, although small amounts of invertebrates were also recovered. Like the menhaden, these species were probably assimilating mostly invertebrate biomass, with refractory detrital components being excreted. Most of the mullet we captured and analyzed were juveniles, whereas most of the gizzard shad were adult size classes. In reservoirs, gizzard shad have been shown to feed mostly on zooplankton when these are abundant (Yako et al. 1996). The grass shrimp was another species that had a large difference in trophic levels estimated by the 2 methods. Again, this species consumed mostly detritus and was consequently assigned a lower trophic level based on diet. Apparently, most of this detritus is refractory, having been ingested along with microorganisms that are the principal nutritional resource.

The concordance of trophic position estimates from the 2 methods for most species gives confidence that the trophic fractionation value 3.3 provides reasonable approximation of $\delta^{15} \mathrm{~N}$ trophic enrichment, despite the fact that trophic $\mathrm{N}$ fractionation can vary in relation to diet protein content and other factors (McCutchan et al. 2003). A likely source of error in the diet-based estimates was bias in the values assigned to invertivorous species that consumed large volumes of detritus. These species were among the most abundant in the marsh, and thus were important in the diets of piscivores. Another potential source of bias in this comparison was the 'time averaging' of assimilated material in isotopic analysis (O'Reilly et al. 2002). Our isotopic data were based on samples collected during August (except for plant tissues, which were collected during August and February, and for which values were averaged). Species with short life cycles can show large temporal variation in isotopic signatures, whereas longer-lived species will reflect a time-integrated record of material assimilation. Thus, our isotopic data may have reflected differing amounts of time integration that depended on body size and other ecological and physiological factors, whereas our dietary samples reflected numerical averages based on available specimens collected over the course of an 18 mo field survey. In most cases, the sizes of specimens used for isotopic analysis closely matched the average size of conspecifics used for dietary analyses. An additional factor that can increase variation in isotopic data is fish movement (Herzka 2005). New immigrants may reflect 
a history of feeding in the habitat from which they emigrated rather than the receiving habitat (Jackson \& Harkness 1987). Given the large potential for differences in time and place to influence variation in diets and isotopic signatures, it is notable that the 2 methods yielded estimates that matched closely, for most of the species examined.

\section{Methods for descriptive food web research}

Each of the methods used in our study of the MIM food web have limitations that reduce the precision and accuracy of inferred web structure; yet, when applied together, stable isotope and dietary analyses provide a more detailed and accurate model of trophic structure and dynamics, including greater taxonomic, temporal, and spatial resolution. Using both dietary and isotopic data, Harrigan et al. (1989) showed that the basal production sources supporting shrimp and grey snappers Lutjanus griseus in southern Florida mangrove and seagrass ecosystems differed markedly. Hentschel (1998) examined both types of data to demonstrate ontogenetic diet shifts and associated shifts in basal sources for polychaete worms. Using stable isotope analysis, Creach et al. (1997) were able to show that amphipods were feeding mostly on plant detritus, although its origin could not be conclusively determined from analysis of gut contents. Currin et al. (2003) inferred from stable isotope data that mummichogs Fundulus heteroclitus were not assimilating most of the detritus contained in guts. Using both methods, Beaudoin et al. (1999) examined betweenpopulation variation in trophic ecology of northern pike Esox lucius, and showed long-term specialization on invertebrates or fishes by conspecifics within populations. To our knowledge, our study is the second to examine an entire community food web using both stable isotope and dietary analyses, and the first to do so for an estuarine system. Mantel et al. (2004) found that the 2 methods produced similar results, stressing the importance of autochthonous resources for consumers in a tropical stream, and that both methods are needed to resolve trophic positions of omnivores.

Even if isotopic data cannot provide a precise and accurate estimate of consumer-resource interactions, the ordination of species according to stable isotope signatures can be used to distinguish patterns of trophic differentiation. Stable isotope ratios have been used to examine niche partitioning (Bootsman et al. 1996), effects of ecosystem engineers on production sources (Botto et al. 2005), trophic differences between migratory and resident fishes (Weinstein et al. 2000), and patterns of migration (Hansson et al. 1997, Herzka 2005), to name only a few topics. An advantage of the stable isotope approach is that large amounts of data can be collected and analyzed with minimal time and effort compared with dietary analysis. In recent years, the number of contract laboratories for massspectometry of stable isotope ratios has increased and costs have declined. The obvious limitation of the method is that it depends on isotopically distinct sources (e.g. algae vs. $\mathrm{C}_{4}$ macrophytes), and for some questions and systems these will not be present. Analysis of multiple elements can increase resolution in some cases (Phillips 2001).

Diet estimates based on stomach contents analysis require large sample sizes, particularly for species with broad diets and high intraspecific variation. Thus, dietary analysis is time and labor intensive, and also requires considerable taxonomic expertise to identify and quantify food items that are often fragmented or partially digested. On the other hand, large samples that include individuals of different size classes collected over time from different locations permit examination of ecological performance in relation to ontogenetic and environmental factors. Moreover, detailed dietary data lend themselves to quantitative estimation of feeding rates based on models of gut evacuation or bioenergetics, and these estimates are essential for development of dynamic food web models. We advocate using both methods in order to maximize precision and accuracy of estimates. Food web models supported by large amounts of empirical data derived from long-term studies should more accurately predict ecological response to natural and anthropogenic perturbations.

Acknowledgements. The Nature Conservancy of Texas and M. Dumesnil provided access to field sites and facilities at Mad Island Marsh Preserve. We thank A. Arrington, J. Arrington, S. Aydin, Y. Bolek, T. Lantz, H. Lopez, M. Morgan, M. Robertson, S. Tarim, and J. Walther for assistance with field data and specimen collection. W. Neill and J. Calvin provided helpful statistical advice. Funding was provided by the Nature Conservancy of Texas, International Sportfish Fund, and a fellowship from Gaziosmanpasa University to S.A.

\section{LITERATURE CITED}

Adams SM, Kimmel BL, Ploskey GR (1983) Sources of organic carbon for reservoir fish production: a trophic-dynamics analysis. Can J Fish Aquat Sci 40:1480-1495

Akin S, Winemiller KO (2006) Seasonal variation in food web composition and structure in a temperate tidal estuary. Estuaries Coasts 29:552-567

Akin S, Winemiller KO, Gelwick FP (2003) Seasonal and spatial variation in fish and macrocrustacean assemblage structure in Mad Island Marsh estuary, Texas. Estuar Coast Shelf Sci 56:1-14

Baird D, Ulanowicz RE (1989) The seasonal dynamics of the Chesapeake Bay ecosystem. Ecol Monogr 59:329-364 
Beaudoin CP, Tonn WM, Prepas EE, Wassenaar LI (1999) Individual specialization and trophic adaptability of northern pike (Esox lucius): an isotope and dietary analysis. Oecologia 120:386-396

Berlow EL, Neutel AM, Cohen JE, de Ruiter PC and 10 others (2004) Interaction strengths in food webs: issues and opportunities. J Anim Ecol 73:585-598

Bootsma HA, Hecky RE, Hesslein RH, Turner GF (1996) Food partitioning among Lake Malawi nearshore fishes as revealed by stable isotope analyses. Ecology 77:1286-1290

Boschker HT, de Brouwer JFC, Cappenberg TE (1999) The contribution of macrophyte-derived organic matter to microbial biomass in salt-marsh sediments: stable carbon isotope analysis of microbial biomarkers. Limnol Oceanogr 44:309-319

Botto F, Valiela I, Iribarne O, Martinetto P, Alberti J (2005) Impact of burrowing crabs on $\mathrm{C}$ and $\mathrm{N}$ sources, control, and transformations in sediments and food webs of SW Atlantic estuaries. Mar Ecol Prog Ser 293:155-164

Bouillon S, Raman AV, Dauby P, Dehairs F (2002) Carbon and nitrogen stable isotope ratios of subtidal benthic invertebrates in an estuarine mangrove ecosystem (Andhra Pradesh, India). Estuar Coast Shelf Sci 54:901-913

Canuel EA, Cloern JE, Ringelberg DB, Guckert JB, Rau GH (1995) Molecular and isotopic tracers used to examine sources of organic-matter and its incorporation into the food webs of San-Francisco Bay. Limnol Oceanogr 40: $67-81$

Christensen V, Pauly D (1992) Ecopath II - a software for balancing steady-state ecosystem models and calculating network characteristics. Ecol Model 61:169-185

Coffin RB, Fry B, Peterson BJ, Wright RT (1989) Carbon isotopic compositions of estuarine bacteria. Limnol Oceanogr 34:1305-1310

Cohen JE, Briand F, Newman CM (1990) Community food webs: data and theory. Springer-Verlag, New York

Connolly RM, Hindell JS, Gorman D (2005) Seagrass and epiphytic algae support nutrition of a fisheries species, Sillago schombergkoii, in adjacent intertidal habitats. Mar Ecol Prog Ser 286:69-79

Cook PLM, Revill AT, Clementson LA, Volkman JK (2004) Carbon and nitrogen cycling on intertidal mudflats of a temperate Australian estuary. III. Sources of organic matter. Mar Ecol Prog Ser 280:55-72

Creach V, Schricke MT, Bertru G, Mariotti A (1997) Stable isotopes and gut analyses to determine feeding relationships in saltmarsh macroconsumers. Estuar Coast Shelf Sci 44:599-611

Currin CA, Newell SY, Paerl HW (1995) The role of standing dead Spartina alterniflora and benthic microalgae in salt marsh food webs: considerations based on multiple stable isotope analysis. Mar Ecol Prog Ser 121:99-116

Currin CA, Wainright SC, Able KW, Weinstein MP, Fuller CM (2003) Determination of food web support and trophic position of the mummichog, Fundulus heteroclitus, in New Jersey smooth cordgrass (Spartina alterniflora), common reed (Phragmites australis), and restored salt marshes. Estuaries 26:495-510

Darnell RM (1961) Trophic spectrum of an estuarine community based on studies of Lake Pontchartrain, Louisiana. Ecology 42:553-568

Deegan LA, Garritt RH (1997) Evidence for spatial variability in estuarine food webs. Mar Ecol Prog Ser 147:31-47

DeNiro MJ, Epstein S (1981) Influence of diet on the distribution of nitrogen isotopes in animals. Geochim Cosmochim Acta 45:341-351

Diener RA, Inglis A, Adams GB (1974) Stomach contents of fishes from clear lake and tributary waters, a Texas estuarine area. Contrib Mar Sci 18:7-17

Divita R, Creel M, Sheridan PF (1983) Foods of coastal fishes during brown shrimp, Penaeus aztecus, migration from Texas estuaries. US Fish Wildl Serv Fish Bull 81:396-402

Fry B, Sherr EB (1984) $\delta^{13} \mathrm{C}$ measurements as indicators of carbon flow in marine and freshwater ecosystems. Contrib Mar Sci 27:13-47

Fry B, Scalan RS, Parker PL (1977) Stable carbon isotope evidence for two sources of organic matter in coastal sediments: seagrasses and plankton. Geochim Cosmochim Acta 41:1875-1877

Garcia de Leon FJ, Gonzalez-Garcia L, Herrera-Castillo JM, Winemiller KO, Banda-Valdes A (2001) Ecology of the alligator gar, Atractosteus spatula, in the Vincente Guerrero Reservoir, Tamaulipas, Mexico. Southwest Nat 46: 151-157

Gelwick FP, Akin S, Arrington DA, Winemiller KO (2001) Seasonal dynamics in abiotic factors and distribution of fishes in the Mad Island Marsh Preserve, Texas. Estuaries 24:285-296

Haines EB, Montague CL (1979) Food sources of estuarine invertebrates analyzed using ${ }^{13} \mathrm{C} /{ }^{12} \mathrm{C}$ ratios. Ecology 60 : 48-56

Hansson S, Hobbie JE, Elmgren R, Larsson U, Fry B, Johannson $\mathrm{S}$ (1997) The stable nitrogen isotope ratios as a marker of food-web interactions and fish migration. Ecology 78 : 2249-2257

Harrigan P, Zieman JC, Macko SA (1989) The base of nutritional support for the gray snapper (Lutjanus griseus): an evaluation based on a combined stomach content and stable isotope analysis. Bull Mar Sci 44:65-77

Hentschel BT (1998) Intraspecific variations in $813 \mathrm{C}$ indicate ontogenetic diet changes in deposit-feeding polychaetes. Ecology 79:1357-1370

Herzka SZ (2005) Assessing connectivity of estuarine fishes based on stable isotope ratio analysis. Estuar Coast Shelf Sci 64:58-69

Jackson D, Harkness DD (1987) The use and interpretation of $\delta{ }^{13} \mathrm{C}$ values as a means of establishing dietary composition. Oikos 48:258-264

Jepsen DB, Winemiller KO (2002) Structure of tropical river food webs revealed by stable isotope ratios. Oikos 96: 46-55

Kwak TJ, Zedler JB (1997) Food web analysis of southern California coastal wetlands using multiple stable isotopes. Oecologia 110:262-277

Manickchand-Heileman S, Arregun-Sanchez F, LaraDominguez A, Soto LA (1998) Energy flow and network analysis of Terminos Lagoon, SW Gulf of Mexico. J Fish Biol 53(A):179-197

Mantel SK, Salas M, Dudgeon D (2004) Food web structure in a tropical forest stream. J North Am Benthol Soc 23: 728-755

Marinucci AC (1982) Trophic importance of Spartina alterniflora production and decomposition to the marshestuarine ecosystem. Biol Conserv 22:35-38

Matlock GC, Garcia MA (1983) Stomach contents of selected fishes from Texas Bay. Contrib Mar Sci 26:95-110

McCutchan JH Jr, Lewis WM Jr, Kendall C, McGrath CC (2003) Variation in trophic shift for stable isotope ratios of carbon, nitrogen, and sulfur. Oikos 102:378-390

Melville AJ, Connolly RM (2003) Spatial analysis of stable isotope data to determine primary sources of nutrition for fish. Oecologia 136:499-507

Minagawa M, Wada E (1984) Stepwise enrichment of $15 \mathrm{~N}$ along food chains: further evidence and the relation 
between $15 \mathrm{~N}$ and animal age. Geochim Cosmochim Acta 48:1135-1140

Odum WE, Zieman JC, Heald EJ (1973) The importance of vascular plant detritus to estuaries. In: Chabreck RH (ed) Coastal marsh and estuary management, Louisiana State University Press, Baton Rouge, LA, p 91-114

O'Reilly CM, Hecky RE, Cohen AS, Plisnier PD (2002) Interpreting stable isotopes in food webs: recognizing the role of time averaging at different trophic levels. Limnol Oceanogr 47:306-309

Peterson BJ, Fry B (1987) Stable isotopes in ecosystem studies. Annu Rev Ecol Syst 18:293-320

Peterson BJ, Howarth RW (1987) Sulfur, carbon, and nitrogen isotopes used to trace organic matter flow in the saltmarsh estuaries of Sapelo Island, Georgia. Limnol Oceanogr 32:1195-1213

Phillips DL (2001) Mixing models in analyses of diet using multiple stable isotopes: a critique. Oecologia 127: 166-170

Phillips DL, Gregg JW (2003) Source partitioning using stable isotopes: coping with too many sources. Oecologia 136: 261-269

Post DM (2002) Using stable isotopes to estimate trophic position: models, methods, and assumptions. Ecology 83: 703-718

Scharf FS, Schlicht KK (2000) Feeding habits of red drum (Sciaenops ocellatus) in Galveston Bay, Texas: seasonal diet variation and predator-prey size relationships. Estuaries 23:128-139

Sheridan PF (1978) Food habits of bay anchovy (Anchoa mitchilli) in Apalachicola Bay, Florida. Northeast Gulf Sci. 2:126-132

Silliman BR, Zieman JC (2001) Top-down control of Spartina alterniflora production by periwinkle grazing in a Virginia salt marsh. Ecology 82:2830-2845

Smith BN, Epstein S (1971) Two categories of ${ }^{13} \mathrm{C} /{ }^{12} \mathrm{C}$ ratios

Editorial responsibility: Kenneth Heck (Contributing Editor), Dauphin Island, Alabama, USA for higher plants. Plant Physiol 47:380-382

Sobczak WV, Cloern JE, Jassby AD, Müller-Solger AB (2002) Bioavailability of organic matter in a highly disturbed estuary: the role of detrital and algal resources. Proc Natl Acad Sci USA 99:8181-8105

Sullivan MJ, Moncreiff CA (1990) Edaphic algae are an important component of salt marsh food-webs: evidence from multiple stable isotope analyses. Mar Ecol Prog Ser 62:149-159

Vander Zanden MJ, Rasmussen JB (1996) A trophic position model of pelagic food webs: impact on contaminant bioaccumulation in lake trout. Ecol Monogr 66:451-477

Vander Zanden MJ, Rasmussen JB (1999) Primary consumer $\delta^{13} \mathrm{C}$ and $\delta^{15} \mathrm{~N}$ and the trophic position of aquatic consumers. Ecology 80:1395-1404

Weinstein MP, Litvin SY, Bosley KL, Fuller CM, Wainwright SC (2000) The role of tidal salt marsh as an energy sources for marine transient and resident finfishes: a stable isotope approach. Trans Am Fish Soc 129:797-810

Winemiller KO (1990) Spatial and temporal variation in tropical fish trophic networks. Ecol Monogr 60:331-367

Winemiller KO, Layman CA (2005) Food web science: moving on the path from abstraction to prediction. In: de Ruiter PC, Wolters V, Moore JC (eds) Dynamic food webs: multispecies assemblages, ecosystem development and environmental change. Elsevier, Amsterdam, p 10-23

Winemiller KO, Polis GA (1996) Food webs: What can they tell us about the world? In: Polis GA, Winemiller KO (eds) Food webs: integration of patterns and dynamics. Chapman \& Hall, New York, p 1-22

Yako LA, Dettmers JM, Stein RA (1996) Feeding preferences of omnivorous gizzard shad as influenced by fish size and zooplankton density. Trans Am Fish Soc 125:753-759

Yodzis P (1998) Local trophodynamics and the interaction of marine mammals and fisheries in the Benguela ecosystem. J Anim Ecol 67:635-658

Submitted: August 2, 2005; Accepted: January 8, 2007

Proofs received from author(s): July 19, 2007 